\title{
A broadband microstrip to waveguide transition for FR4 multilayer PCBs up to 50 GHz.
}

\author{
Carlo Buoli, Vito Marco Gadaleta, Tommaso Turillo, Alessandro Zingirian \\ Siemens Information and Communication Networks S.p.A \\ Strada Padana Superiore Km158 20060 Cassina de' Pecchi (MI) - ITALY \\ Telephone: +390295266340 Fax: +390295266375 e-mail: Carlo.Buoli@icn.siemens.it
}

\begin{abstract}
A broadband microstrip-to-waveguide transition has been studied and tested for low-cost microwave circuits on FR4 multilayer boards having an internal thick metal plate (1). It is obtained, at first, milling a slot in a thick metal plate. Subsequently the PCB is built up, using standard PCB process, inserting the thick metal plate as the second layer. In this way the slot results filled with FR4 prepreg. A further simple milling process removes the lower layers up to the thick metal plate and creates a part of the waveguide transformer and an area for plate to metal housing connection. The transition integration in the multilayer is obtained just fixing the board on the metal housing which includes the rest of the transformer and the waveguide. Moreover, as the transition shows low sensitivity to manufacturing tolerances, the above process is characterised by high reliability and reproducibility.
\end{abstract}

\section{INTRODUCTION}

Recent microwave transceiver design is moving towards low cost manufacturing (2), fully automated assembly and high production volumes. A multilayer PCB having a thick metal plate as an internal layer is particularly suitable to satisfy these features (3). In fact it allows to integrate microwave circuits (both high power and low noise) with IF, control and power supply circuits and can be obtained using the standard PCB process. To build a complete transmitter and/or receiver on such a structure a microstrip to waveguide transition is needed. The proposed solution is particularly suitable for the mentioned kind of board: in facts it results from the assembly process itself, without requiring any other specific interventions. Electromagnetic simulations have been performed and measures confirm low insertion losses and broadband performance due to the introduction of a waveguide transformer (4) (5).

\section{THE STRUCTURE}

It is possible to design microwave circuits on a low-cost FR4 multilayer PCB using the structure shown in Figure 1. Microstrips are on the first metal layer [met1], while the second metal layer [met2] is a thick metal plate. It acts as electrical ground and heat sink for microwave active devices and offers a stiff support to the upper thin FR4 layer [diel1]; additional circuits (bias, control signals, IF paths) can be housed on the third metal layer [met3] while the fourth metal layer [met4] is a further ground plane. If necessary, low losses circuits, built on alumina substrate, can be welded on the thick metal plate after creating an opening on the first dielectric layer [diel1], and they are connected to the other circuits by means of wire-bonds. A similar procedure can be used to mount high power components: in this case the metal plate [met2] works as a flange.

In order to integrate a complete transceiver on a single board in a fully automated way and at low cost, it is necessary to introduce in such a structure a microstrip to waveguide transition without increasing fabrication complexity and not exceeding FR4 multilayer standard processes. The transition we present in this paper meets 
these goals, resulting directly from the board fabrication process.

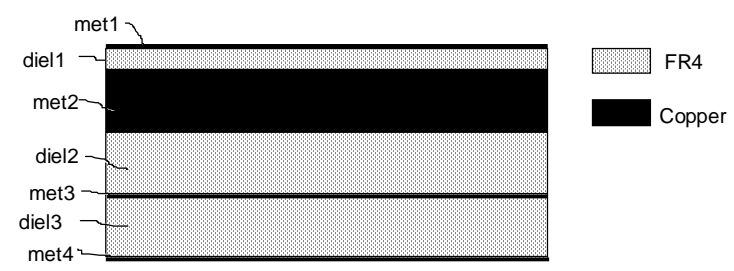

Figure 1: Multilayer PCB having a thick copper plate as the second layer.

\section{DESCRIPTION OF THE TRANSITION}

The proposed transition consists of the following elements (see figure 2):

Patch: the microstrip ends with a metal patch [1] whose shape and position have been chosen to match the coupling between the microstrip [2] and the waveguide [3], over the desired frequency band.

Backshort: a metal backshort [4] covering the last part of the microstrip has been used as a reflector and to prevent the propagation on air.

Slot [5]: it is milled in the thick metal plate [met2] and filled with FR4. Its length and width have been chosen to make the transition work in the desired frequency band.

Waveguide transformer: it is a rectangular waveguide obtained part [6] in the thick metal plate and part [7] in the metal housing [8] to match the slot to the waveguide below.

Waveguide: it is directly dug in the metal housing.

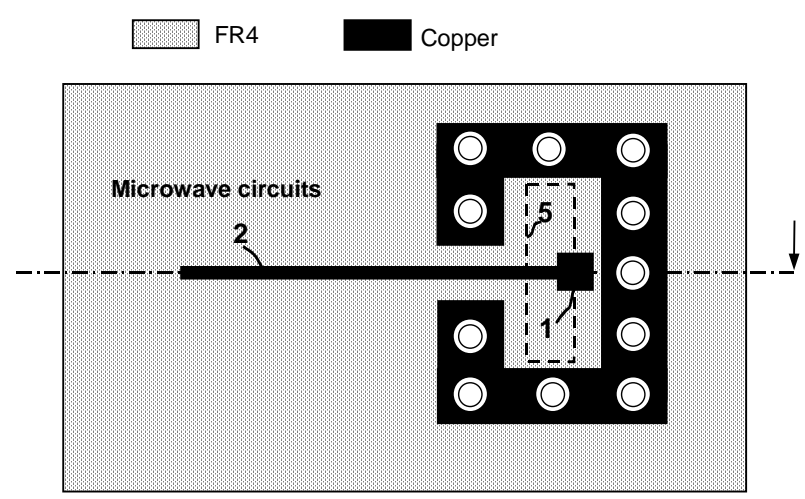

(Figure2a)

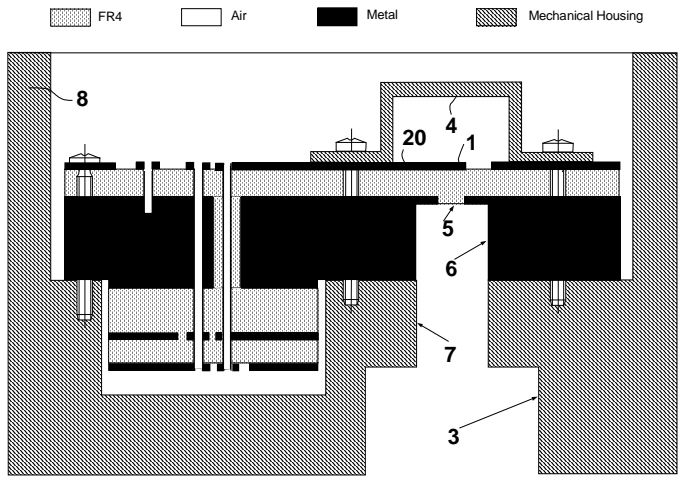

(Figure2b)

Figure 2: Integration of the proposed transition in the FR4 multilayer board having a thick metal plate as a second layer. Top view (a) and side view (b).

These are the fabrication steps (see Figure 1 and Figure 2):

1. The slot [5] is milled in the thick metal plate.

2. The PCB is built up inserting the metal plate as the second metal layer [met2]; in this way the slot results filled with FR4 prepreg.

3. A second milling process removes the lower layers [met4, diel3, met3, diel2] up to the thick metal plate smoothing its surface to make it clean and ready to be joined to the metal housing [8].

4. A third milling process creates part of the waveguide transformer [6] on the thick metal plate and reduces the thickness of the slot.

5. The waveguide [3] and the other part of the transformer [7] are embedded in the metal housing during its fabrication process.

The described steps don't require critical processes and the transition is automatically achieved just screwing the board on the metal housing, without further operations like device mounting or bonding.

\section{RESULTS}

We performed several electromagnetic simulations to evaluate the optimal EM-field distribution in the structure. The microstrip to waveguide transition proposed shows very good performance in terms of bandwidth and losses. Simulation results also show how the use of the waveguide transformer can improve the working relative bandwidth up to $20 \%$.

A transition covering both the $27.5-29.5 \mathrm{GHz}$ and 31.8 $33.4 \mathrm{GHz}$ band has been developed. The board is an FR4 four-layer having an internal metal plate $1 \mathrm{~mm}$ thick. Microstrip circuits lie on the upper 100 micron thick dielectric layer. Simulation results (see Figure 3a) show a 
return loss better than $20 \mathrm{~dB}$ and low losses $(0.5 \mathrm{~dB})$ over the frequency band of interest (see Figure $3 b$ ).

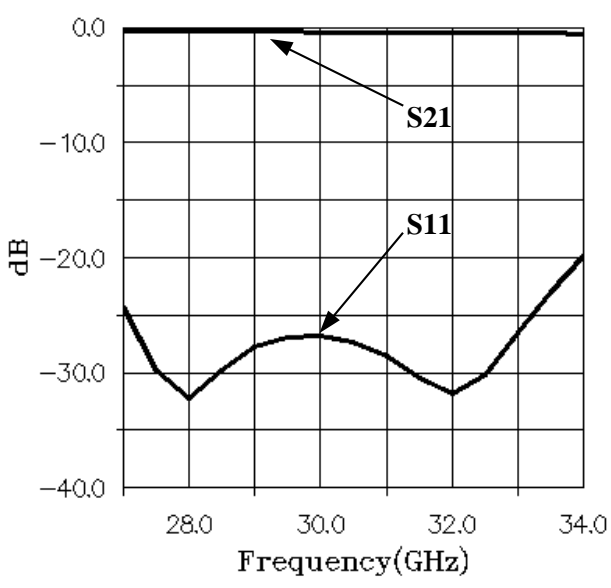

(a)

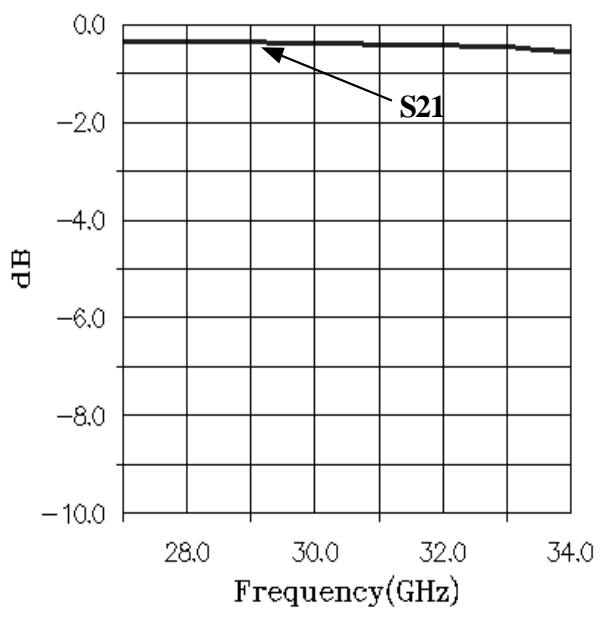

(b)

Figure 3: Results of an Agilent HFSS ${ }^{T M}$ electromagnetic simulation for a transition covering both the 27.5-29.5 GHz and 31.8-33.4GHz bands: (a) insertion and return losses; (b) detail of insertion losses.

Such a microstrip to waveguide transition has been tested in a $28 \mathrm{GHz} \mathrm{Rx} / \mathrm{Tx}$ (see Figure 4,5,6). To evaluate the transition performance a coaxial cable has been soldered to the microstrip line just before the transition. The measures are shown on Figure 7. Measures of saturated power of the transmitter and noise figure of the receiver confirmed low insertion loss of the transition. Figure 8 shows good match between simulation and measure results.

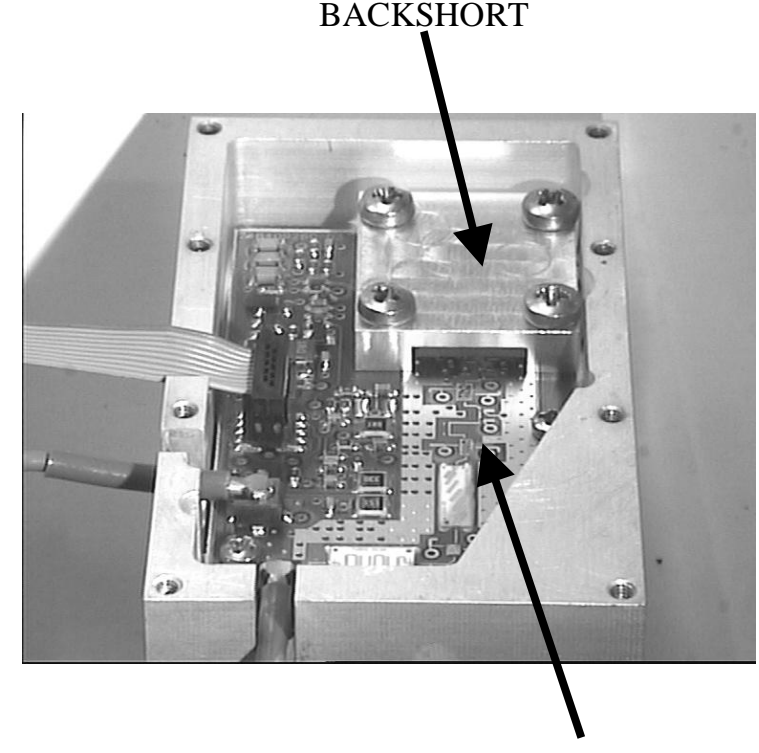

MICROSTRIPS

Figure 4 : Complete $28 \mathrm{GHz}$ transmitter including the discussed transition, covered by the backshort

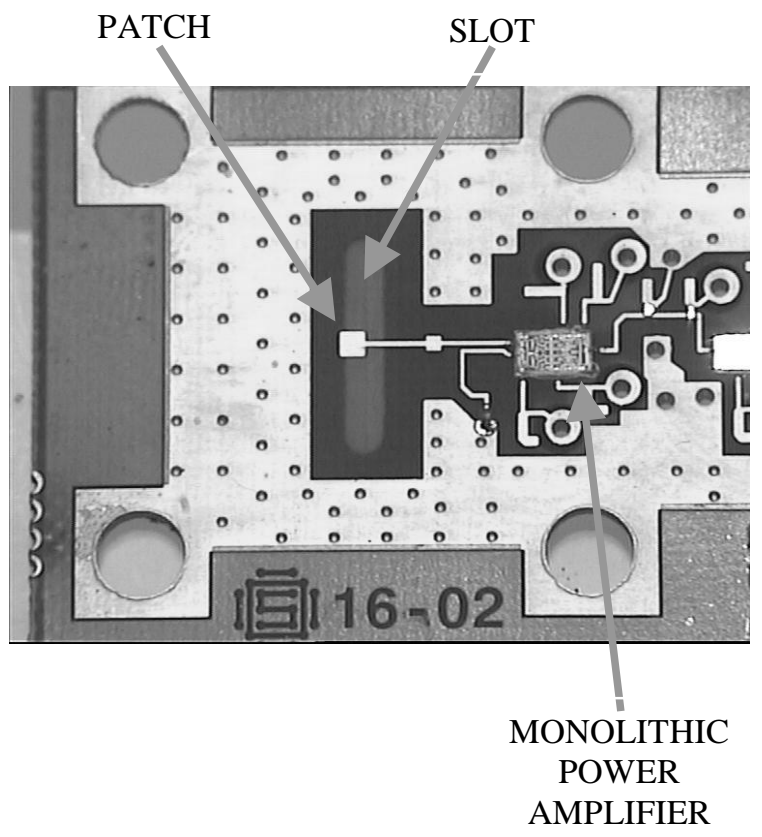

Figure 5: On the upper layer of the transition the microstrip ends with a square patch 


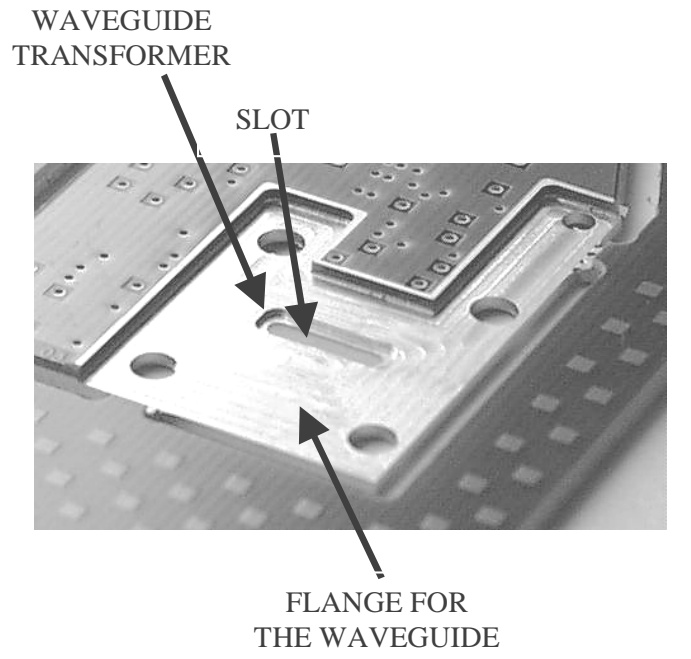

Figure 6: This bottom view of the board shows the slot, part of the waveguide transformer and the clean copper plate

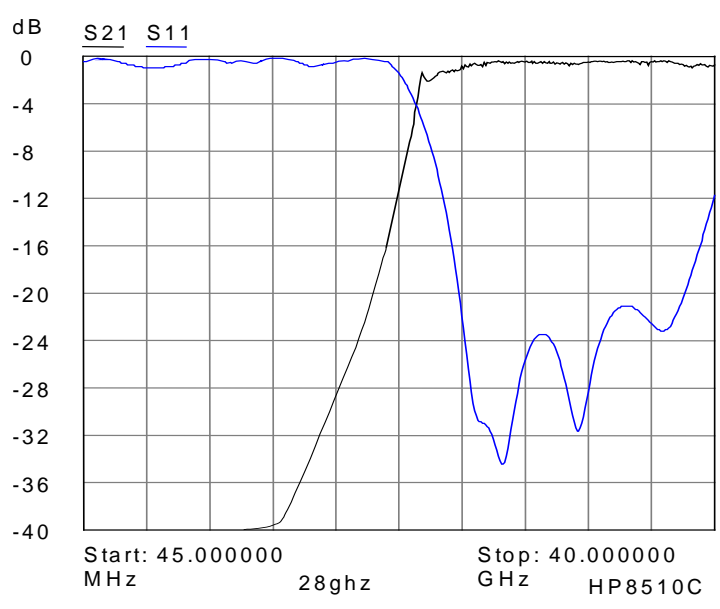

Figure 7: Measured scattering parameters for the transition of the $28 \mathrm{GHz}$, transmitter emphasise broadband performance.

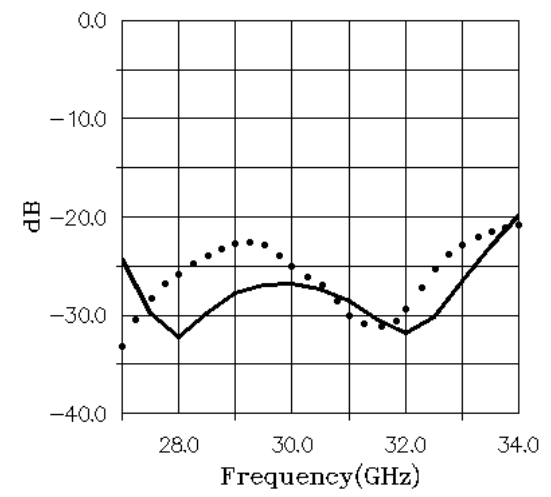

Figure 8: Comparison between simulated (solid line) and measured (dotted line) return loss of the transition in the band of interest.

\section{CONCLUSIONS}

A broadband microstrip-to-waveguide transition has been proposed. It is obtained on a FR4 multilayer having an internal metal plate whose thickness guarantees good stiffness, heat dissipation and electrical ground. Completely integrated in the multilayer and metal housing, the transition doesn't require any manual intervention and it is not affected by FR4 dielectric losses. Finally the measures show a very wide band: therefore no limitation to microstrip circuits bandwidth are introduced. This features make the proposed transition the right solution for integrating on the same substrate whole receivers/transmitters characterised by fully automated assembly, low costs of manufacturing and high production volumes.

\section{REFERENCES}

(1) Patent pending

$$
\text { C. Buoli, G. Mora, L.A. Cervi, FR4 PCB }
$$

Modulation Transfer MWVCO Up to 16 GHz, Sept.1997, 27th European Microwave Conf., Jerusalem, Israel

(3)

C.Buoli , G. Biffi, T. Turillo, A.Zingirian, Thick metal plate insertion make FR4 multilayer board a simple carrier for RF power circuits, 31 th European Microwave Conf., London, UK, 23-26 September 2001 Antennas, 1995, Artech House, chapter 2, pp.19-41

Robert E. Collin, Foundation for Microwave Engineering, $2^{\text {nd }}$ ed., 1992, McGraw-Hill International Edition, Chapter 5: Impedance transformation and matching 